\section{Military Technical College Kobry El-kobbah, Cairo, Egypt}

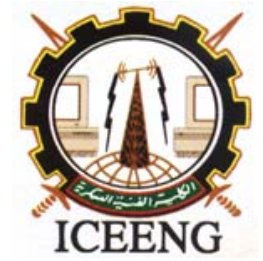

\author{
$5^{\text {th }}$ International Conference \\ on Electrical Engineering \\ ICEENG 2006
}

\title{
An Assessment between Classical Fuzzy and Fuzzy Model Reference Learning Controller Design of Electro-Optical pointing and tracking System
}

\author{
G. Elnashar
}

\author{
T. Elbayoumi \\ Egyptian Armed Forces
}

M. Hegazy

\begin{abstract}
Electro-optical pointing and tracking systems (EOPTS) have a wide range of military and civilian applications. The passive line of sight (LOS) stabilization systems are multi-input multi-output (MIMO) systems that are highly nonlinear and possess a strong coupling effect between their states. it presents a challenging systems to control. In this paper the analysis of the passive (EOPTS) stabilization system with the development of its nonlinear models is derived. Two different types of control algorithms are presented. The first controller is a classical fuzzy control. The controller presents a good technique that proves to be stable with high transient and tracking performances. The controller is applied to the LOS stabilization system and the simulation results are introduced. Next, A Model Reference Learning fuzzy Controller less dependant on the designer knowledge of the LOS stabilization system is proposed. Such controller possesses a learning mechanism that is able to form its rule-base by watching the system behavior. The learning mechanism utilizes a reference model that describes the desired performance. The designed process of the controller and simulation results of implementing the controller to the system is introduced. Finally, comparative analysis between the two developed controllers is conducted. It discusses the advantages and disadvantages of each controller algorithm.
\end{abstract}

Keywords: Multi input multi output (MIMO), fuzzy control, Fuzzy Model reference learning control (FMRLC), LOS stabilized system.

\section{FORMULATION OF SYSTEM EQUATIONS}

Figure (1) shows a gyro stabilized platform system [1,2]. There are generally three main components, a flywheel, motors and mirror system. Two gimbals that provide two-degree of freedom to the flywheel, Inner gimbal provides movement along the yaw axis and outer gimbal provides movement along the pitch axis, two torque motors are used to control the pitch axis and the yaw axis. A mirror that is geared to the inner gimbals through a 2:1 reduction drive mechanism. Figure (2) shows schematic diagram of the gyro-mirror LOS system. The LOS stabilization system consists of four main modules, namely the rotor $(\mathrm{R})$, the inner gimbal (IG), the outer gimbal (OG) and the mirror (Mr). The coordinate frames and the moment of inertia (MI) of each element along the principle axes are defined as follows:

$i$ index of three dimensional axis $(1,2,3) . \quad v_{i}$ vehicle frame/system frame, assumed fixed

$A$ MI of outer gimbal (OG) about $g_{1}, \quad B, C, D$ MI of inner gimbal (IG) about $r_{1}, r_{2}$ and $r_{3}$ $E, F, G$ MI of mirror about $m_{1}, m_{2}$ and $m_{3} \quad g_{i}$ outer gimbal frame

$r_{i} \quad$ inner gimbal and rotor frame, $m_{i}$ mirror frame

$H \quad$ MI of rotor (R) about $r_{1}$ or $r_{2} \quad J \quad$ MI of rotor (R) about $r_{3}$ 


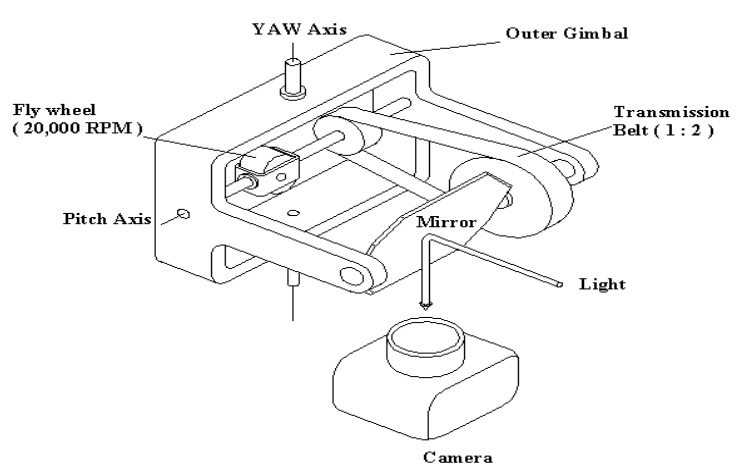

Fig.1 A passive Gyro-stabilized platform

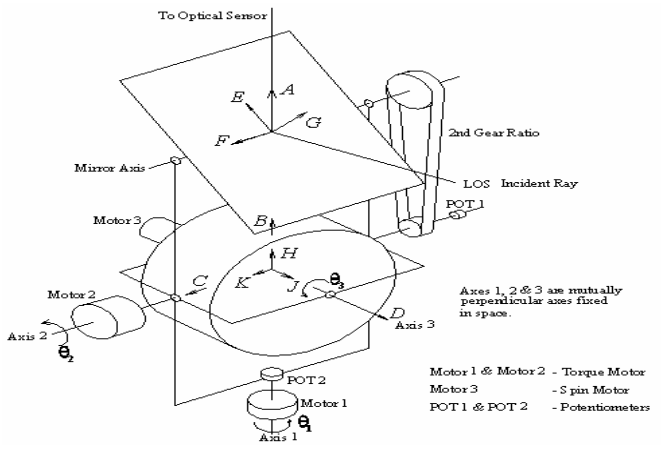

Fig. 2 Schematic of passive LOS stabilization system

As shown in Figure 2, the gyro mirror LOS platform has two coordinate axis (yaw axis and pitch axis), one tracking pointer (mirror) and a flywheel. By defining the coordinate frame. The transformation matrices between the coordinate frames are given by:

$$
\begin{aligned}
& {\left[\begin{array}{l}
g_{1} \\
g_{2} \\
g_{3}
\end{array}\right]=\left[\begin{array}{ccc}
1 & 0 & 0 \\
0 & \cos \theta_{1} & \sin \theta_{1} \\
0 & -\sin \theta_{1} & \cos \theta_{1}
\end{array}\right]\left[\begin{array}{l}
v_{1} \\
v_{2} \\
v_{3}
\end{array}\right]} \\
& {\left[\begin{array}{l}
r_{1} \\
r_{2} \\
r_{3}
\end{array}\right]=\left[\begin{array}{ccc}
\cos \theta_{2} & 0 & -\sin \theta_{2} \\
0 & 1 & \sin \theta_{1} \\
\sin \theta_{2} & 0 & \cos \theta_{2}
\end{array}\right]\left[\begin{array}{l}
g_{1} \\
g_{2} \\
g_{3}
\end{array}\right]} \\
& {\left[\begin{array}{l}
m_{1} \\
m_{2} \\
m_{3}
\end{array}\right]=\left[\begin{array}{ccc}
\cos \left(\frac{\theta_{2}}{2}+\frac{\pi}{4}\right) & 0 & -\sin \left(\frac{\theta_{2}}{2}+\frac{\pi}{4}\right) \\
\sin \left(\frac{\theta_{2}}{2}+\frac{\pi}{4}\right) & 0 & \cos \left(\frac{\theta_{2}}{2}+\frac{\pi}{4}\right)
\end{array}\right]\left[\begin{array}{l}
g_{1} \\
g_{2} \\
g_{3}
\end{array}\right]}
\end{aligned}
$$

Where $\theta_{1}$ and $\theta_{2}$ are angle of rotation about axis 1 and 2 respectively as shown in Figure (1$2)$. The angular velocities of the mechanical elements are as follows:

$$
\begin{aligned}
& \Omega_{R}=\dot{\theta}_{1} g_{1}+\dot{\theta}_{2} r_{2}+\dot{\theta}_{3} r_{3} \\
& \Omega_{I G}=\dot{\theta}_{1} g_{1}+\dot{\theta}_{2} r_{2} \\
& \Omega_{O G}=\dot{\theta}_{1} g_{1} \\
& \Omega_{M r}=\dot{\theta}_{1} g_{1}+\frac{1}{2} \dot{\theta}_{2} m_{2}
\end{aligned}
$$

where $\Omega_{R}, \Omega_{I G}, \Omega_{O G}$ and $\Omega_{M r}$ are the angular velocities of the rotor, inner gimbal, outer gimbal and the mirror respectively. Using the transformation matrices shown above, and its corrseponding own coordinate frame and define the rotational kinetic energy for the system as a rigid body. The kinetic energy simplifies to a sum of three terms that is given by [3]:

$$
K_{e}=\sum_{i=1}^{3} \frac{1}{2} I_{i} \Omega_{i}^{2}
$$

Where $i=1,2,3$ are the principle axes of each frame. Therefore, the kinetic energy of the elements are 


$$
\begin{aligned}
& K_{e}=K_{e_{O G}}+K_{e_{I G}}+K_{e_{M r}}+K_{e_{R}} \\
& =\frac{1}{2} A \dot{\theta}_{1}^{2}+\frac{1}{2} B \dot{\theta}_{1}^{2} \cos ^{2} \theta_{2}+\frac{1}{2} C \dot{\theta}_{2}^{2}+\frac{1}{2} D \dot{\theta}_{1}^{2} \sin ^{2} \theta_{2}+\frac{1}{2} E \dot{\theta}_{1}^{2} \cos ^{2} x+\frac{1}{2}\left(\frac{F}{4}\right) \dot{\theta}_{2}^{2}+ \\
& +\frac{1}{2} G \dot{\theta}_{1}^{2} \sin ^{2} x+\frac{1}{2} H\left(\dot{\theta}_{1}^{2} \cos \theta_{2}+\dot{\theta}_{2}^{2}\right)+\frac{1}{2} J\left(\dot{\theta}_{1} \sin \theta_{2}+\dot{\theta}_{3}\right)^{2}
\end{aligned}
$$

Assuming that the system is rigid enough such that the strain energies are negligible, the Lagrange's equations thus become[4]:

$\frac{d}{d t}\left(\frac{\partial K_{e}}{\partial \dot{q_{i}}}\right)-\frac{\partial K_{e}}{\partial q_{i}}=Q_{i}$

Where

$K_{e}$ denotes the kinetic energy, $q_{i}$ denotes a generalized coordinate $\left(\theta_{1}, \theta_{2}\right.$ and $\left.\theta_{3}\right)$, and

$Q_{i}$ denotes a generalized force $\tau_{1}$ and $\tau_{2}$.

Applying Lagrange's equation (10) to equation (9) we obtain:

$$
\begin{aligned}
& (A+D) \ddot{\theta}_{1}+(B-D+H) \ddot{\theta}_{1} \cos ^{2} \theta_{2}+\frac{1}{2}(E+G) \ddot{\theta}_{1}+\frac{1}{2}(G-E) \dot{\theta}_{1} \dot{\theta}_{2} \cos \theta_{2}+J \omega_{s} \dot{\theta}_{2} \cos \theta_{2}=\tau_{1} \\
& \left(C+\frac{F}{4}+H\right) \ddot{\theta}_{2}+\frac{1}{2}(B-D+H) \dot{\theta}_{1}^{2} \sin 2 \theta_{2}-\frac{1}{4}(G-E) \dot{\theta}_{1}^{2} \cos \theta_{2}-J \omega_{s} \dot{\theta}_{1} \cos \theta_{2}=\tau_{2}
\end{aligned}
$$

where $\omega_{s}=\theta_{3}+\theta 1 \sin \theta_{2}$ is the rotor spin velocity. It is a constant angular velocity of the flywheel.Equations (11) and (12) represent the nonlinear model of the LOS stabilization system., the following properties of the LOS system may be inferred as follow [4]:

Property I: The terms coefficient of $\ddot{\theta}_{1}$ and $\ddot{\theta}_{2}$ are positive definite. This is an essential property of the system. This property will be used later in the development of controllers.

Property II: The cross-couplings between the axes due to $\theta_{2}$ terms appearing in equation (11) and $\theta 1$ terms appearing in (12). The magnitudes of these values are small, and thus the crosscoupling effects are week. However, the inclusion of the flywheel introduces strong crosscoupling between the axes of the system as can be seen from the presence of $\theta_{3}$ in the last terms of equations (11) and (12). $\theta_{3}$ is usually in the order of thousand rpm. This strong crosscoupling increases the difficulty of the control problem. For the passive LOS stabilization system, the control requirement can be stated as: achieve a sufficient high bandwidth with no steady state error for step inputs and decouple the system such that there is minimal crosscoupling effect in the system. The ability of the proposed control to meet the above requirement will be considered in the following sections. Define the state variable, $X$, control signal, $U$, and the output vector , Y, as follows:

$$
X=\left[\begin{array}{llll}
\dot{\theta_{1}} & \dot{\theta_{2}} & \theta_{1} & \theta_{2}
\end{array}\right]^{T}, U=\left[\begin{array}{ll}
\tau_{1} & \tau_{2}
\end{array}\right]^{T} \text {, and } Y=\left[\begin{array}{ll}
\theta_{1} & \theta_{2}
\end{array}\right]^{T}
$$

\section{DESIGNING REQUIREMENT}

The ultimate requirement to the compensator is, that it works "well" for real system. This requirement can be subdivided into the following four categories:

- Nominal stability: The compensator must ensure internal stability in the controlled system, provided the model is correct

- Nominal Performance: The compensator must minimize the error

- Robust Stability: for all models the compensator must ensure internal stability

- Robust performance: for all models the compensator must ensure that the error is within a specified bound 


\section{FUZZY CONTROL}

Fuzzy system theory was first introduced to the research community in 1965 by Zadah [5]. Fuzzy set theory can be considered as a development of the classical set theory. In his fuzzy theory, Zadah assumes a gradual transition from one set to another. Accordingly, better presentation of different variable can be obtained with minimal number of sets. Hence classical sets are a simplified case of fuzzy sets where sets the membership level takes only two values, zero or one.

\subsection{Limitation of Conventional Controllers}

Conventional controllers can not used in all applications because it has a lot of restrictions:

(1) Plant nonlinearity: Nonlinear models are computational intensive and have complex stability problem.(2) Plant uncertainty: A plant does not have accurate models due to uncertainty and lack of prefect knowledge. (3) Uncertainty in measurements and difficult to model. However Fuzzy Control is used in different research and industrial due to its advantages:(1)Ability to translate imprecise /vague knowledge of human experts. (2) Smooth and robust controller behavior.

\subsection{Fuzzy Control Structure}

Fuzzy control theory can be found in many text books a and papers [6-8]. However the controller is composed of four elements as follow:

(1) Fuzzyfication interface: it converts the crisp inputs to linguistic values that are easy to manipulate through controller's components.

(2)Rule-base: It is a set of If-then rules that describes the knowledge of the experts of how to control the process.

(3)Inference mechanism: It is mechanism that uses the fuzzified inputs together with the rulebase to form the fuzzy control action.

(4)Defuzzification interface: it converts the fuzzy conclusion into a crisp value suitable to be used as an input to the process.

\subsection{Classical LOS Fuzzy Control}

A full matrix fuzzy controller is designed to control the two state variables $\left(\theta_{1}, \theta_{2}\right)$ of the LOS system considering the strong coupling effect the system possesses. Two fuzzy controllers are used as direct controllers (forward path between $\theta_{1}$ $\tau_{1}$ and $\theta_{2}-\tau_{2}$ while the other two controllers are used to decouple the cross relationship between $\theta_{1}-\tau_{1}$ and $\theta_{2}-\tau_{2}$. Figure (3) shows the controller structure

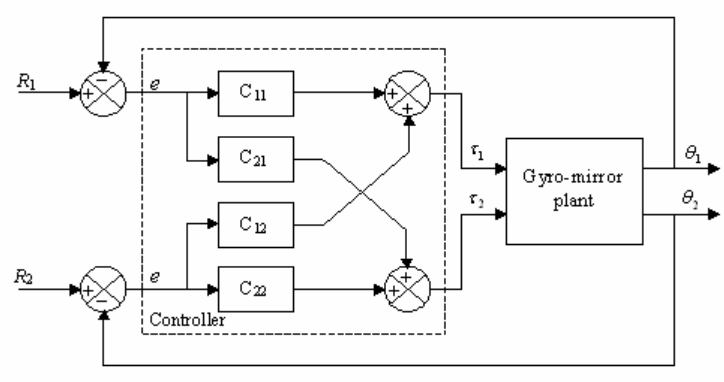

Fig.3 Full matrix fuzzy controller block diagram

The four controllers are MISO fuzzy controllers with two inputs representing the error and the change rate of the error. The inputs are given by the following equations.

$e_{\theta_{1}}(K T)=\dot{\theta}_{1}(K T)-\theta_{1}(K T), \quad c_{\theta_{1}}(K T)=\frac{e_{\theta_{1}}(K T)-e_{\theta_{1}}((K-1) T)}{T}$

$e_{\theta 2}(K T)=\dot{\theta}_{2}(K T)-\theta_{2}(K T), \quad c_{\theta 2}(K T)=\frac{e_{\theta 21}(K T)-e_{\theta 2}((K-1) T)}{T}$

Where $e, c$ represent the error and the change rate of error respectively and $\stackrel{*}{\theta}, \theta$ represent the desired and measured angles respectively. Five membership functions are used for each 
input and output of the four fuzzy controllers. Larger number of membership functions would not enhance the controller performance dramatically; however it will increase the complication of the design process. On the other hand, fewer number of the membership functions will significantly affect the controller performance negatively. Skewed triangular membership functions are used for inputs of the four fuzzy controllers.

For the outputs, singletone membership functions are used. The singletone membership function simplifies the defuzzification when used for crisp outputs. Finally, the rules at rulebase of the fuzzy controller were formed by examining the linearized model of the LOS system in addition to the open loop analysis; the rule base was formed according to the following facts:

The coupling effect dominates the characteristics of the system; hence, to move one gimbal, the controller is required to generate a sufficiently high torque about the gimbal perpendicular axis rather than its axis[6,9]. Appling a positive torque to the yaw channel will vary both angles positively. Meanwhile, a positive torque in the pitch channel implies a negative variation in the yaw angle and positive one in the pitch angle. The rule base was constructed so that it represents a human expert in the loop. For instance, one rule that a human may use to control the system is "if the pitch angle is less than the set point ( $e_{\theta 2}$ is positive) then $\tau_{1}$ should be positive" an other rule that would represent more detailed information is "if that angle is less than the set point and approaching that point very fast a negative torque may be applied to make shore that we don't over shoot the set point. The rule base is indicated in Table (1). Full matrix fuzzy controller was implemented to the LOS system. The system performance was satisfactory; however, the performance is not guaranteed to be optimal. This is due to the fact that the controller parameters were set based on the designer knowledge about the system behavior.

\begin{tabular}{|c|c|c|c|c|c|}
\hline 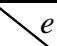 & $\mathrm{NB}$ & NS & $\mathrm{ZE}$ & PS & PB \\
\hline NB & PS & PB & NB & $\mathrm{ZE}$ & PS \\
\hline $\mathrm{NS}$ & $\mathrm{NS}$ & NB & NB & PB & PS \\
\hline $\mathrm{ZE}$ & PS & $\mathrm{PO}$ & $\mathrm{ZE}$ & PB & NS \\
\hline PS & $\mathrm{NS}$ & $\mathrm{ZE}$ & PS & $\mathrm{PB}$ & $\mathrm{NB}$ \\
\hline PB & PB & $\mathrm{ZE}$ & PB & PS & $\mathrm{NB}$ \\
\hline
\end{tabular}

Rule base for the yaw angle direct controller $\mathrm{C} 11$

\begin{tabular}{|c|c|c|c|c|c|}
\hline 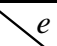 & NB & $\mathrm{NS}$ & $\mathrm{ZE}$ & PS & PB \\
\hline NB & NS & PB & PB & PS & NS \\
\hline NS & $\mathrm{PS}$ & $\mathrm{PB}$ & PS & NB & $\mathrm{NS}$ \\
\hline $\mathrm{ZE}$ & NB & PS & $\mathrm{ZE}$ & $\mathrm{NS}$ & PS \\
\hline $\mathrm{PS}$ & PB & $\mathrm{PB}$ & $\mathrm{NS}$ & $\mathrm{ZE}$ & PB \\
\hline PB & NB & $\mathrm{PB}$ & $\mathrm{NS}$ & $\mathrm{NS}$ & PB \\
\hline
\end{tabular}

Rule base for the yaw angle direct controller $\mathrm{C} 22$

\begin{tabular}{|l|l|l|l|l|l|}
\hline $\begin{array}{l}\text { c } e \\
\text { c }\end{array}$ & NB & NS & ZE & PS & PB \\
\hline NB & PS & PS & NB & NS & PS \\
\hline NS & NS & ZE & NS & ZE & PS \\
\hline ZE & PB & PS & ZE & NS & NS \\
\hline PS & NB & PB & PS & PB & NB \\
\hline PB & PB & PB & PS & NS & NB \\
\hline
\end{tabular}

Rule base for the yaw angle direct controller $\mathrm{C} 21$

\begin{tabular}{|l|l|l|l|l|l|}
\hline $\begin{array}{l}\text { e } \\
c\end{array}$ & NB & NS & ZE & PS & PB \\
\hline NB & NS & NS & PS & PS & NS \\
\hline NS & PS & PB & ZE & PS & NS \\
\hline ZE & NB & NB & ZE & PS & PS \\
\hline PS & PB & ZE & NS & PS & PB \\
\hline PB & NB & NB & NS & PS & PB \\
\hline
\end{tabular}

Rule base for the yaw angle direct controller $\mathrm{C} 12$

Table 1: Rule-bases of the full matrix fuzzy controller for the LOS stabilization system (NB: Negative big, NS: Negative, ZE: Zero, PS: Positive, PB: Positive big)

\section{FMRL CONTROLLER STRUCTURE}

A Multi-Input Multi-Output Fuzzy Model Reference Controller (MIMOFMRLC) is proposed. The controller provides the non-linear control surface that compensates for system nonlinearity and decouples the interaction between its two channels. Fuzzy model reference control adopts the same principles of Model Reference Adaptive Control (MRAC) [7] that proved stability and high capabilities of disturbance rejection. It was first introduced as a Self 
Organizing Fuzzy Controller (SOFC) by Procky and Mamdani [10]. Pasino et. al. developed a simplified algorithm and was referred to as Fuzzy model reference learning control. The term learning verifies the fact that algorithm incorporates a memory that store and recall the control behavior. The algorithm was implemented for various types of plant and shows high transient and tracking performances. The structure of the FMRLC is as shown in Figure (4). It consists of plant model (passive LOS stabilization system), fuzzy controller, reference model, and learning mechanism. The controller is responsible of providing the appropriate control action to follow the desired set point. The learning mechanism is composed of inverse model and knowledge base modifier. The learning mechanism achieves the following: Observes data from a fuzzy control system [i.e. $y_{r}(k T)$ and $\left.y(k T)\right]$. Characterizes its current performance. Automatically synthesizes and/or adjusts the fuzzy controller so that some specified performance objectives are met. These performance objectives are characterized via the reference model. The inverse model describes the inverse characteristics of the plant. It provides the learning mechanism with preliminary information about how to modify the control action to force the system to follow the performance given by the reference model. The knowledge base modifier seeks the necessary modification of the controller knowledgebase (rules of the fuzzy controller) based on the information supplied by the inverse model. This modification forces the system to follow the desired set point with the performance that matches the one given by the reference model the desired performance can be described for both channels by means of reference model. A two-input, two-output learning fuzzy controller was used to provide fast adaptation and learning capabilities. This assures stable system with a high transient performance. A complicated learning algorithm would slow down the learning process and hence, reduces the stability especially for system with high dynamics [11-13]. Figure (4) shows the structure of the controller The two controllers have the same inputs. The inputs represent the error in the yaw angle $\left(e_{\theta_{1}}\right)$ and the error in the pitch angle $\left(e_{\theta_{2}}\right)$. It is possible to include the first derivative of $e_{\theta_{1}}$ and $e_{\theta_{2}}$ to the controller inputs to reduce the oscillation around the set point. To minimize this oscillation, a derivative control action should be included by adding the derivative of the errors $e_{\theta_{1}}$ and $e_{\theta_{2}}$ to the inputs of the fuzzy controller. For two input fuzzy system, if five memberships functions are assigned for each input, the maximum number of rules would be 25 rules (all possible combination of the inputs), However, for four inputs fuzzy system, the rules at the rule-base would be 625 rules. Hence, the required learning time would increase exponentially and reduces the controller performance. To reduce the oscillation yet keep learning simple, a conventional derivative controller was added to the main fuzzy controller. It is assumed that the adaptation process is able to compensate for the error between the non linearity of the $1^{\text {st }}$ order dynamics of the system and the linearity of the derivative controller. The outputs of controllers are the torques of the two motors ( $\tau 1$ and $\tau 2$ respectively). The controller inputs are given by:

$e_{\theta_{1}}(k T)=\theta_{1}{ }^{*}(k T)-\theta_{1}(k T), \quad e_{\theta_{2}}(k T)=\theta_{2}{ }^{*}(k T)-\theta_{2}(k T)$

Where $\theta_{1}{ }^{*}(k T), \theta_{2}{ }^{*}(k T), \theta_{1}(k T)$, and $\theta_{2}(k T)$ represent the desired yaw angle, the desired pitch angle, the measured yaw angle and measured pitch angle respectively. Although the inputs are the same, each implicit controller (within the MIMO fuzzy controller) has independent input scaling factors. The gains, $g \theta_{1} e_{\theta 1}, g \theta_{1} e_{\theta 2}$ represent input scaling gains of the yaw angle controller for the yaw angle error and the pitch angle error respectively. Similarly $g \theta_{2} e_{\theta 1}$ and $g \theta_{2} e_{\theta 2}$ are the input scaling gains of the pitch angle controller for the yaw angle and the pitch angle errors respectively. Triangular membership functions for both inputs and outputs universes of discourse, Zadeh's compositional rule of inference and COG Defuzzification were implemented for the fuzzy controllers and the inverse models. 


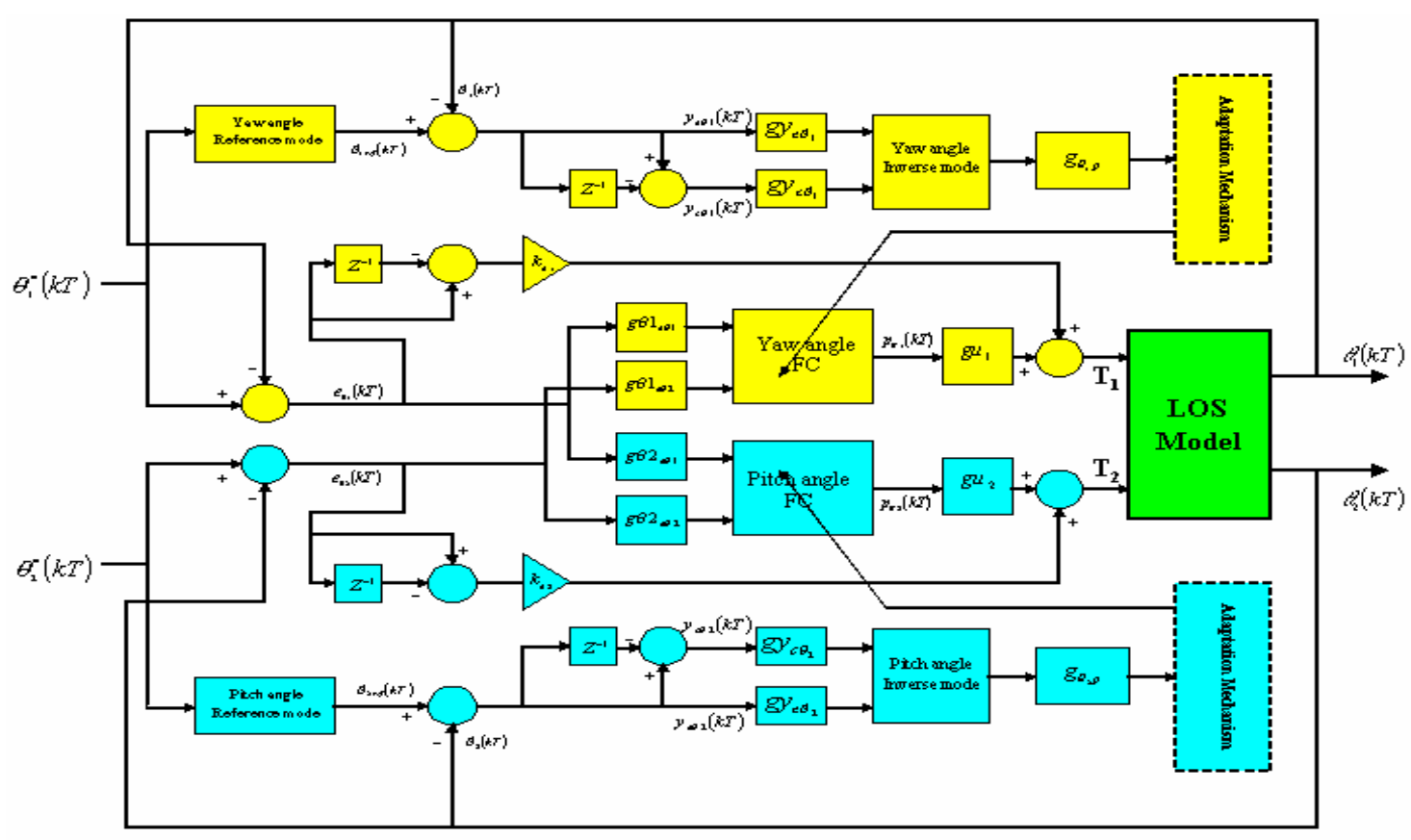

Fig. (4) MIMO FMRLC structure

\subsection{Adaptation Mechanism}

The centers of the output membership function for each controller are subjected to the adaptation. The centers of the output membership functions for the yaw angle controller and the pitch angle controller are given by:

$$
b_{\theta_{1} i}(k T)=b_{\theta_{1}}(k T-T)+g_{\theta_{2} p} p_{\theta_{2}}(k T), \quad b_{\theta_{2} i}(k T)=b_{\theta_{2} i}(k T-T)+g_{\theta_{1} p} p_{\theta 1}(k T)
$$

where $g_{\theta_{1} p}, g_{\theta_{2} p}, p_{\theta_{1}}(k T)$, and $p_{\theta_{2}}(k T)$ are the adaptation gain of the yaw angle, the adaptation gain of the pitch angle, the output of the yaw angle inverse model, and the output of the pitch angle inverse model respectively. The inverse model of the yaw and pitch channels are fuzzy inverse models representing the inverse dynamics of the yaw and the pitch angles respectively. Each inverse model was designed separately assuming only the coupling relationship between the state variables $\theta_{1}, \theta_{2}$ and the control actions $\left(\tau_{1}, \tau_{2}\right)$. The rule-base for the two inverse models are carefully designed. The inverse model inputs are given by

$$
\begin{array}{ll}
y_{e \theta_{1}}(k)=\theta_{1 r e f}(k T)-\theta_{1}(k T), & y_{c \theta_{1}}(k)=\frac{y e_{\theta_{1}}(k T)-y e_{\theta_{1}}(k T-T)}{T} \\
y_{e \theta_{2}}(k)=\theta_{2 \text { ref }}(k T)-\theta_{2}(k T), & y_{c \theta_{2}}(k)=\frac{y e_{\theta_{2}}(k T)-y e_{\theta_{2}}(k T-T)}{T}
\end{array}
$$

where $\theta_{1 \text { ref }}, \theta_{1}, \theta_{2 \text { ref }}$ and $\theta_{2}$ are the output of the yaw reference model, the measured yaw angle, the output of the pitch reference model, and the output of the pitch angle model respectively. The reference model for each controller was selected according to the required performance of both channels taking into consideration the system limitations (maximum torques and system parameters). To avoid generating control actions exceeding the torque limits of the system the following equations were added [11]:

$$
\begin{array}{lc}
b_{\theta 1 i}(k T)=\tau_{\min } \text { if } b_{\theta 1 i}(k T) \leq \tau_{\min }, & b_{\theta 1 i}(k T)=\tau_{\max } \text { if } b_{\theta 1 i}(k T) \geq \tau_{\max } \\
b_{\theta 2 i}(k T)=\tau_{\min } \text { if } b_{\theta 2 i}(k T) \leq \tau_{\min } & b_{\theta 2 i}(k T)=\tau_{\max } \text { if } b_{\theta 2 i}(k T) \geq \tau_{\max }
\end{array}
$$

where $b_{\theta 1 i}(k T)$ and $b_{\theta 2 i}(k T)$ are the centers of the output membership functions of the yaw angle and the pitch angle respectively, $\tau_{\min }, \tau_{\max }$ are the minimum and maximum torque of 
the two motors respectively. The parameter of the controller were selected using trial and error however, some guidelines were used to reduce the time effort spent on obtaining the appropriate values of these parameters. The reference model is a linear second order system, with parameters representing desired performance and satisfies the system limitations. Uniform distributed triangular membership functions were used for the input and output. The rule base was formed through the knowledge of the system behavior. The learning coefficient is a critical factor to the system stability and performance. There is tradeoff between fast learning capability and system stability with minimum oscillation at steady state. Large learning coefficient enhances transient performance and adapt faster to parameter variations. However, system is sensitive to small error at steady state and as a result oscillation behavior occurs. Small learning coefficient provides performance at steady state however; slower learning and transient performance are expected.

\section{SIMULATION RESULTS}

A prototype for the passive LOS stabilization system has the parameters as follows: $\mathrm{A}\left(\mathrm{Kgm}^{2}\right)=0.0392, \mathrm{~B}\left(\mathrm{Kgm}^{2}\right)=0.0211, \mathrm{C}\left(\mathrm{Kgm}^{2}\right)=0.0153, \mathrm{D}\left(\mathrm{Kgm}^{2}\right)=0.0049, \mathrm{E}\left(\mathrm{Kgm}^{2}\right)$ $=0.0019, \mathrm{~F}\left(\mathrm{Kgm}^{2}\right)=0.0018, \mathrm{G}\left(\mathrm{Kgm}^{2}\right)=0.0036, \mathrm{H}\left(\mathrm{Kgm}^{2}\right)=0.0057, \mathrm{~J}\left(\mathrm{Kgm}^{2}\right)=0.0089$, $\omega_{\mathrm{s}}(\mathrm{rad} / \mathrm{s})=800$.

Series of rectangular pulses was applied to the LOS system as the desired angles in both yaw and pitch angles. The frequencies of the inputs were set differently to examine the coupling effect at different points. The maximum desired deviation angles were set to 0.1 radians. Figure (5) Phase plane of LOS system using full matrix fuzzy controller. The phase plane in Figure (5) and table(2) shows that the system has a high coupling effect (see subplots c, d, e, and f) where a variaton in one channel developed oscillation in the opposite channel. It can be noted that the system is stable with satisfactory performance. It can be noted that the direct controller produces the torque in the direction of the desired angle. In addition, the coupling controller provides a torque in the opposite channel that aids the direct one to move the gimbale to the desired angle. The opposite coupling controller compensates the disturbance in the opposite channel due to coupling effect. In general, that fuzzy controller provides stability to the system with minimum overshoot and fast transient for both channels. It also reduces the coupling effect between the two channels. The control action is smooth and minimal Despite of the satisfactory results, it is not guaranteed that the results are optimal. This is due the ambiguity of the coupling behavior and the design process that doesn't use optimal design tools. Figure (6) and table (2) shows how the learning mechanism was able to form the control surface and to find the decoupling relation between the system states inputs $\left(\tau_{1}, \tau_{2}\right)$ and output $\theta_{1}$ and $\theta_{2}$. It also shows the development of such learning. The best results of training can be achieved by training the system with inputs similar to the ones that will experience during the use and implementation phase. After the first period of training, system performances enhanced dramatically. Figure (6) represents the phase plan of the yaw and pitch channels respectively when step changes applied to both channels simultaneously. Figure $(6 \mathrm{c}, \mathrm{d})$ shows the coupling effect on yaw channel when step change is applied to pitch channel and vise versa in Figure (6 e,f). It can be noted that the control algorithm provides an asymptotically stable system that approaches the equilibrium point in all cases. The coupling effect is minimal even when applying simultaneous changes in both channels. For performance comparison purposes and system qualification. They are: (1) Integral absolute error $\left(\mathrm{IAE}_{i}\right)$ is used to evaluate the system tracking performance (2) Integral square error $\left(\mathrm{ISE}_{i}\right.$ ) is similar to (IAE) however it discriminates between systems that have close (IAE). (3) Integral time multiple Absolute error $\left(\mathrm{ITAE}_{i}\right)$ is used to evaluate system performance with the time. (4) Integral absolute control action $\left(\mathrm{IACA}_{i}\right)$ is used to evaluate the efficiency of the 
system. Table (2) shows that better results were achieved using the FMRLC especially after sufficient learning period. Also by reviewing the IAE and ISE of the fuzzy controller, they show that FMRLC gives much better performances

\section{CONCLUSION}

A comparative study between the two control algorithms that are designed for the LOS system is presented. The study shows that the MIMOFLC provides a more stable system with less sensitivity to the coupling effect. It also shows that the MIMOFMRLC is more efficient and has a higher performance than the other classical fuzzy algorithms. Fuzzy model reference learning controller is similar to the fuzzy controller except, it has two additional advantages, (1) it is less dependant on the designer knowledge of the system where the rulesbase of the controller is formed using learning mechanisms. (2) Useful information about the controller and hence the system can be achieved after learning through reading the formed rules. It can be noted that the FMRLC is less complicated than the other two controllers. In fact this conclusion coincides with the fact that conventional control techniques work better for simple linear systems, while fuzzy controllers produce much better results for complex nonlinear systems such as the LOS Stabilization System.

\section{References}

[1] Loh.Ming.Kit. "Design, Development of a LOS Stabilization Systems". Department of Mechanical and production Engineering, National University of Singapore, 1991

[2] C.E.Smith. “Applied Mechanics: More Dynamics”. John Willy \& Sons, 1976.

[3] R.E.J.Swain. "A two degree of freedom gyroscope with frictionless inner and outer gembals pick-offs". Mechanical Technology of Inertial Devices, Proceedings of the Institute of Mechanical engineers, pages 9-16 Newcastle, April 7-9, 1987.

[4] Y.Netzer. "Line-of-sight steering and stabilization”. Optical Engneering, 21(1):96104,Jan-Feb 1982.

[5] Zadeh L.A. "Fuzzy Sets". Information and control 8, 1965, pp. 338-353.

[6] T. H. Lee, J. H. Nie and M. W. Lee "A Fuzzy Controller with Decoupling for Multivariable Nonlinear Servo-Mechanisms, with Application to Real-Time Control of a Passive Line-Of-Sight Stabilization System" Mechatronics, Vol. 7, No. 1, 1997

[7] Layne J. and Passino K., "Fuzzy model reference learning control" In Proc. 1st IEEE Conference Control Applications, Dayton, OH, May/June 1995, pp. 624-632.

[8] E. H. Mamdani, S.Assilian "An experiment in linguistic synthesis with a fuzzy logic controller”. Int.J.Man-Mach.Stud.,Vol.7,No.1,1975,pp.1-13.

[9] A.KRue. "Stabilization of precision electro optical pointing and tracking systems". IEEE Transactions on Aerospace and Electronic Systems, 5(5):805-819, $1969 .$.

[10] Lavne J., Passino K., Yurkvoich S. "Fuzzy model learning control for antiskid braking systems" IEEE Trans. Contr. Syst. Techno., vol. 1, No. 2, June 1993, pp.122-129.

[11] Li Zhen, L. Xu "Fuzzy Learning Enhanced Speed Control of an Indirect Field-Oriented Induction Drive" IEEE Transaction on Control Systems Technology, Vol. 8, No. 2, March 2000, pp.270-278.

[12] Ahmed El Dessouky “Adaptive fuzzy control for high performance induction motor drives" Ph.D. Thesis, Royal Military College of Canada, July 202, pp 117-137 .

[13] A. El Dessouky, M. Tarbouchi “A MIMO Adaptive Fuzzy Controller for Induction Motors" Special Issue of WSEAS Transactions in Systems, 2003 


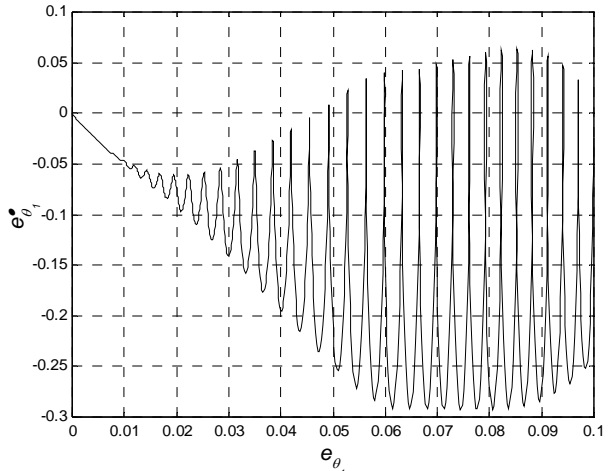

(a)

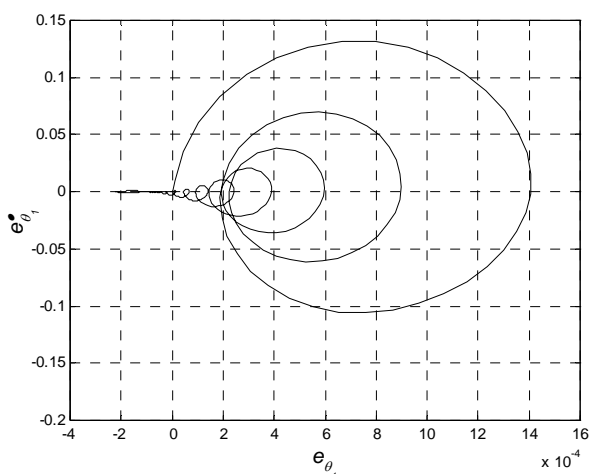

(c)

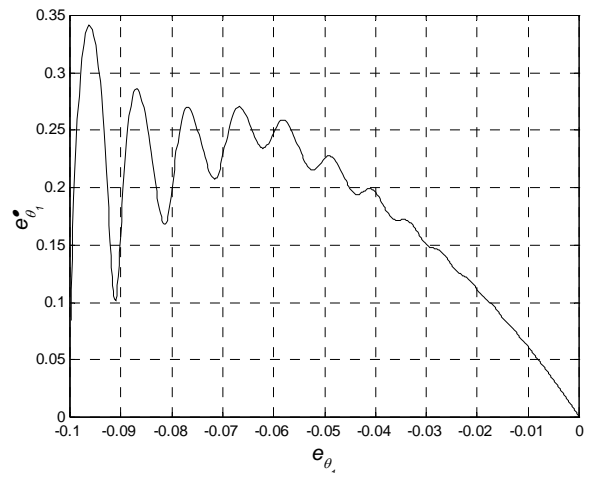

(e)

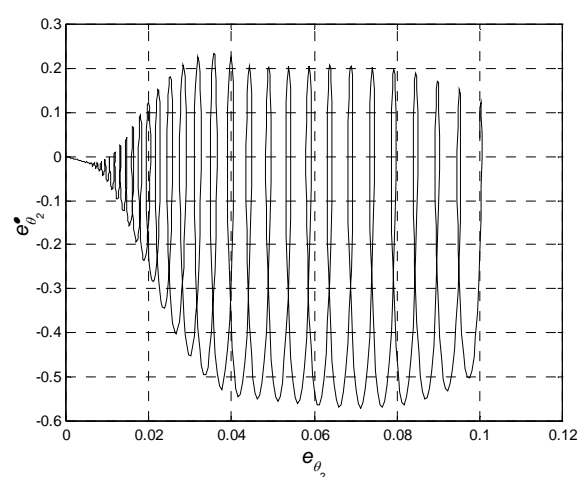

(b)

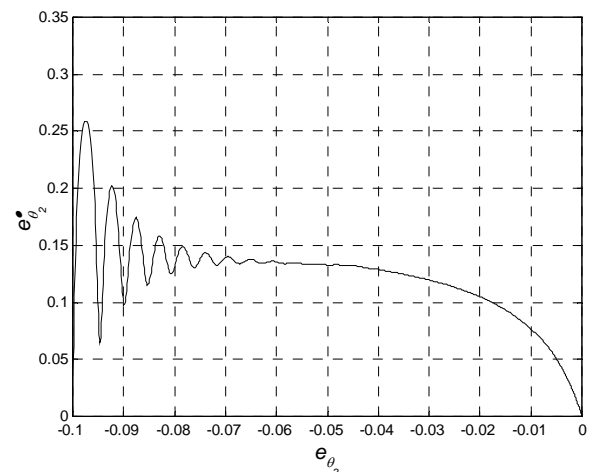

(d)

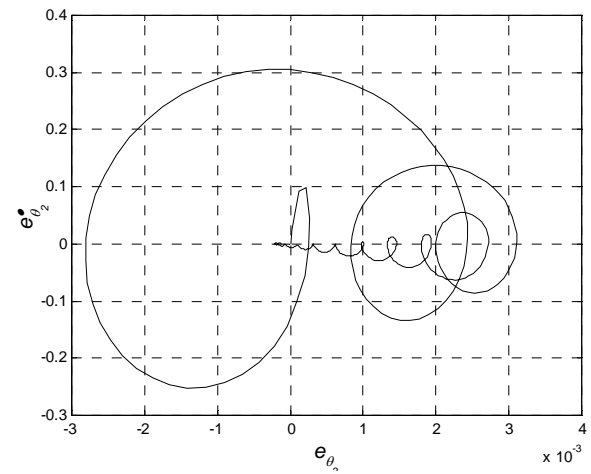

(f)

Figure ( 5) Phase plane of LOS system using full matrix fuzzy controller (a) and (b) for channel one and two respectively when applying step inputs to the two channels simultaneously, (c) coupling effect of channel two on channel one (d) no coupling effect on channel two (e) no coupling effect on channel one (f) coupling effect of channel one on channel two 


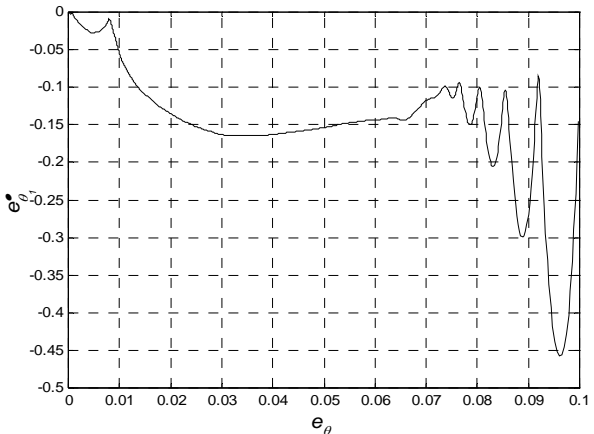

(a)

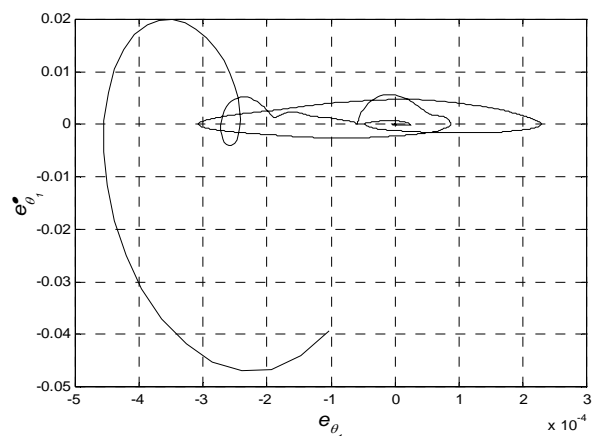

(c)

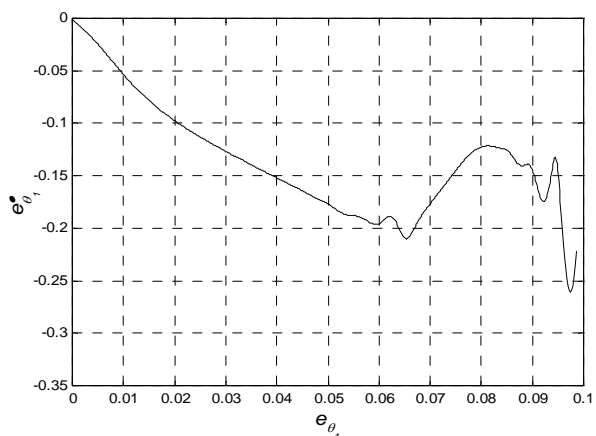

(e)

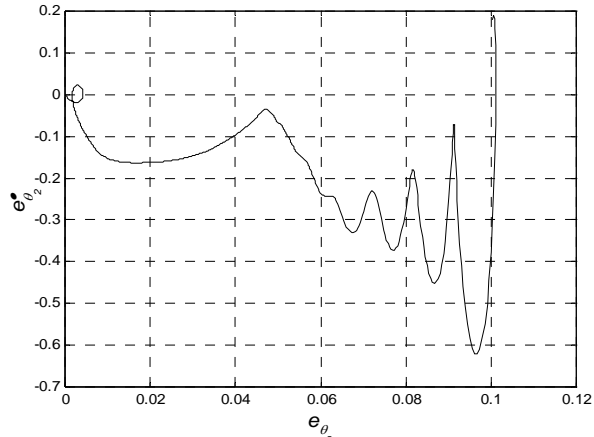

(b)

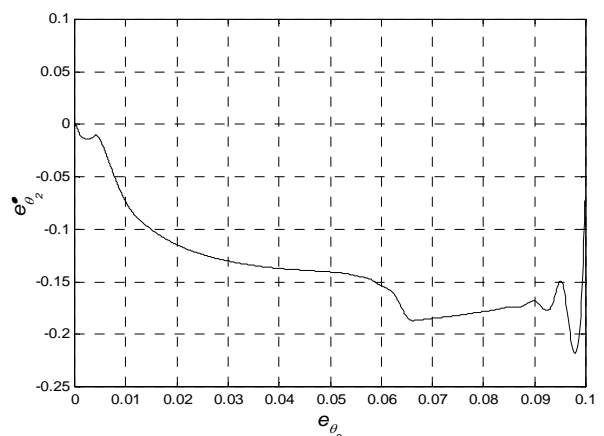

(d)

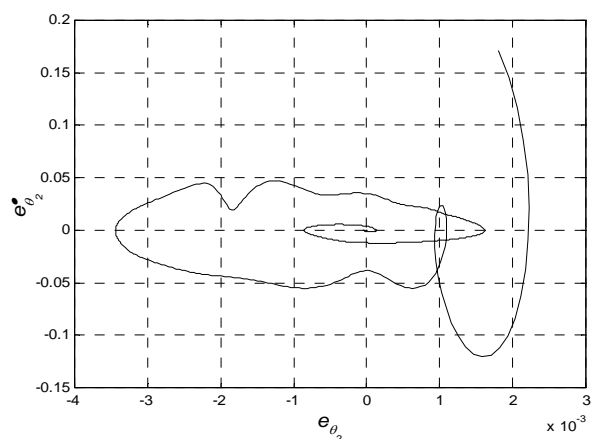

(f)

Figure (6) Phase plane of LOS system using FMRLC (a) and (b) for channel one and two respectively when applying step inputs to the two channels simultaneously, (c) coupling effect of channel two on channel one (d) no coupling effect on channel two (e) no coupling effect on channel one (f) coupling effect of channel one on channel two

\begin{tabular}{|c|c|c|c|c|c|c|c|c|}
\hline \multirow{2}{*}{ Controller } & \multicolumn{2}{|l|}{ IAE } & \multicolumn{2}{|l|}{ ITAE } & \multicolumn{2}{|l|}{ ISE } & \multicolumn{2}{|l|}{ IACA } \\
\hline & Yaw & pitch & yaw & pitch & yaw & pitch & $\tau 1$ & $\tau 2$ \\
\hline $\begin{array}{l}\text { FMRLC after } \\
\text { learning }\end{array}$ & 141.7 & 246 & 1147.3 & 1264.1 & 8.8 & 15.2 & 2929.4 & 5387.6 \\
\hline $\begin{array}{l}\text { FMRLC before } \\
\text { learning }\end{array}$ & 186.4 & 292.5 & 1158.7 & 1432.4 & 12.2 & 18.9 & 3744.7 & 6062.9 \\
\hline Fuzzy controller & 195.3 & 338 & 2746.5 & 3636.8 & 12.2 & 20.6 & 6646.9 & 3681.1 \\
\hline
\end{tabular}

Table 2: Comparison results for pulsed reference signal 